\title{
Stability Properties for Learning with Heterogeneous Expectations and Multiple Equilibria
}

\author{
Eran A. Guse* \\ University of Helsinki \\ Department of Economics, University of Helsinki \\ Discussion Papers, No. 574:2003 \\ ISSN 1459-3696 \\ ISBN 952-10-1224-2
}

August 27, 2003

\begin{abstract}
The bounded rationality literature has studied heterogeneous learning rules under models with a single equilibrium. This paper examines learning with heterogeneous expectations in a simple macroeconomic model with multiple equilibria. Stability properties of this model are determined by the distribution of heterogeneity. These results differ greatly from those which impose homogenous expectations a priori. When the level of heterogeneity is allowed to vary, stability conditions become more restrictive due to the stationarity requirements of the simpler, more parsimonious updating rule. Finally, I find that the two equilibria exchange stability when the MSE of using this simpler updating rule is minimized.

Key Words: Adaptive Learning; Heterogeneous Expectations; Multiple Equilibria; Rational Expectations.
\end{abstract}

JEL Classification: C62, D84, E37

\footnotetext{
* Address correspondence to: Research Unit on Economic Structures and Growth, P.O. Box 10 (Snellmaninkatu 12 B), FIN-00014 University of Helsinki. Phone: +358 9191 24626. Fax: +358-9-191 7980 E-mail: eran.guse@helsinki.fi. This work is part of research at the Research Unit on Economic Structures and Growth. Financial support from the Academy of Finland, Yrjö Jahnsson Foundation, Bank of Finland and Nokia Group is gratefully acknowledged. I would like to thank Shankha Chakraborty, George Evans, and Sunny M.C.Wong for their comments and insights.
} 


\section{Introduction}

Expectations have been an important part of many macroeconomic models. Consumers and firms maximize their expected utility, or profit, based upon their expectations of several economic variables Since they effect the decision process, expectations will naturally also have a large impact on the macroeconomy. The current accepted method for modelling expectations in macroeconomics is allowing agents to form Rational Expectations (RE). With RE, agents form their expectations using the mathematical expectations operator conditioned upon available information usually involving perfect knowledge of the market equilibrium. Although RE is the solution norm for the profession, many economists believe that RE gives agents too much intelligence by assuming that they know all the equilibrium parameter values of the model.

Since the introduction of RE by Muth (1961) and Lucas $(1972,1973)$, the bounded rationality literature has presented several alternatives. The works of Townsend (1978), Bray and Savin (1986), Evans and Honkapohja (2001), Guesnerie (1992), and Hommes and Sorger (1998) have suggested several types of less sophisticated expectations schemes to limit the intelligence of economic agents.

One method to limit hyperrationality is by allowing agents to learn the parameters of the model through time. With econometric learning, agents are boundedly rational with the ability to learn the parameters of the model by using least squares to form their expectations. The agents attempt to estimate the REE by using data from the economy. They form statistical estimates of the equilibrium parameters and update these estimates every period. It has been discovered that the learning process is stable and converges to the rational expectations equilibrium (REE) for some macroeconomic models with reasonable parameter values. Evans (1989) and Evans and Honkapohja (1992) introduced the E-stability principle which states that the mapping from the perceived law of motion (PLM) to the actual law of motion (ALM) governs the stability of the REE under least squares learning. ${ }^{1}$ Using their E-stability principle, for some macroeconomic models, we can determine the stability of the solution under this learning rule. One may think that if these solutions are E-stable, then using RE may not be such an undesirable method to solve for long run equilibria. Furthermore, for models with multiple equilibria, we can distinguish between solutions that are E-stable and those that are not E-stable.

1 For a detailed explanation of E-stability, see Evans and Honkapohja (2001). 
In the learning literature, a few papers consider models with heterogenous expectations. Evans and Honkapohja (1997) focus on a situation where all agents use the same learning process, but they all may begin with different initial expectations. They find that all of the agents will asymptotically learn the RE solution analogous to the RE in the homogeneous expectations case as long as it is E-stable. Evans, Honkapohja, and Marimon (2001) allow for heterogeneity and random adjustments in expectations in a model with a continuum [0,1] agents. They suppose that each agent may have one of two types of expectations given some probability. They find that the heterogeneity disappears asymptotically and the expectations then converge to the RE solution. Under this form of heterogeneity, the stability conditions may not be the same in some models for homogenous and heterogenous expectations. Honkapohja and Mitra (2002) present structural heterogeneity where the representative agent can not be used. They find that this type of heterogeneous learning affects stability conditions for learning for some common macroeconomic models. Giannitsarou (2003) considers three types of heterogeneity: initial perceptions, different degrees of inertia in updating, and different learning rules. She shows that the last two types of heterogeneity may cause different stability conditions than with homogeneous expectations.

The above literature has focused mainly on heterogeneity in models with a single equilibrium. Multiple equilibria has been well discussed in the macroeconomic literature by Azariadis (1981), Cass and Shell (1983), Azariadis and Guesnerie (1986), and Farmer (1999). When heterogeneous expectations exist in a model with multiple equilibria, one can examine how heterogeneity affects the stability properties of more than one equilibrium under learning. Furthermore, if the level of heterogeneity is allowed to change, the stability properties of the equilibria can be further examined.

This paper presents heterogeneity in a different manner than the papers listed above. This is a fourth type of heterogeneity that can exist in a model with multiple equilibria. I assume that there are two types of agents with different perceived laws of motion. The first type believes that some economic variable follows an independent and identically distributed (i.i.d.) process while the second type believes that this variable follows an AR(1) process. Both types of agents use least squares to learn the true process of the economic variable in question. This paper examines how this form of heterogeneity affects E-stability in a specific "ad hoc" macroeconomic model. I show that the E-stability conditions will be affected by the proportion of agents that use each perceived law of motion. Finally, I examine the relationship between E-stability and the mean square error 
(MSE) of each equilibrium. It turns out that the two equilibria "exchange" stability at a level of heterogeneity where the MSE of the inefficient predictor is minimized.

\section{The Model}

\subsection{Homogeneous Expectations}

The model used in this paper is a linear stochastic macroeconomic model. The Sargent and Wallace (1975) "ad hoc" model, an example of one of these types of models, is made up of three equations: an aggregate supply equation, an IS equation and a LM equation. These three equations can be reduced to a univariate reduced form that represents the equilibrium of this model.

Another linear stochastic macroeconomic model is the real balance model of Taylor (1977). This is a variation of the rational expectations IS-LM model where the real balance effect enters each equation. Like the Sargent and Wallace (1975) model, this model can be reduced to a single equation representing the equilibrium. This model has the possibility of multiple stationary REE, and it has previously been the focus of detailed analysis in the learning literature, for example see Evans and Honkapohja (1994) and Heinemann (2000).

The reduced form of these models is the following:

$$
y_{t}=\alpha+\beta_{0} E_{t-1}^{*} y_{t}+\beta_{1} E_{t-1}^{*} y_{t+1}+v_{t}
$$

where $E^{*}$ denotes a not necessarily rational expectation and $v_{t}$ is a linear combination of iid stochastic shocks. Therefore, $E_{t-1} v_{t}=0$.

\section{REE Solutions}

I first present the solutions to the model under homogeneous expectations, so that the impact to the solutions from heterogeneity can be fully understood. Each agent will use their perceived law of motion (PLM) of the economy to estimate the parameters of the model. The solution to the model will depend upon the distribution of the agents with different PLM's, so the solution to equation 1 will depend on the averaged perceived law of motion among all agents. The averaged PLM produces the actual law of motion (ALM) followed by the economy. If all agents use the 
following perceived law of motion (defined as $P L M_{1}$ or the "first predictor"):

$$
y_{t}=a_{1}+v_{t}
$$

then the unique (REE) is the following:

$$
a_{1}=\frac{\alpha}{1-\beta_{0}-\beta_{1}}
$$

If all agents use the following PLM (defined as $P L M_{2}$ or the "second predictor"):

$$
y_{t}=a_{2}+b_{2} y_{t-1}+v_{t}
$$

then there are two REE of the following:

$$
\begin{aligned}
& a_{2}=\frac{-\alpha}{\beta_{1}} \text { and } b_{2}=\frac{1-\beta_{0}}{\beta_{1}} \text { or } \\
& a_{2}=\frac{\alpha}{1-\beta_{0}-\beta_{1}} \text { and } b_{2}=0 .
\end{aligned}
$$

These solutions are referred to as the AR(1) solution and the minimum state variable (MSV) solution respectively. As one can see, the MSV solution to the model using $P L M_{2}$ has the same value as the solution to the model using $P L M_{1}$.

\section{E-stability Conditions}

In order for agents to learn an equilibrium value for the parameters of the model, the equilibrium must be E-stable.

Definition 1: E-stability is the condition of local asymptotic stability of a fixed point $\bar{\phi}=T(\bar{\phi})$ under the differential equation ${ }^{2}$

$$
\frac{d \phi}{d \tau}=T(\phi)-\phi
$$

where $T$ is the mapping from the perceived law of motion, $\phi$, to the implied actual law of motion, $T(\phi)$ and $\tau$ denotes "notional" or "artificial" time.

Formal details of the T-map, $T$, are provided below. If an equilibrium is E-stable, then the agents will asymptotically learn the given equilibrium. This is a local condition, so initial parameter estimates must be in the neighborhood of attraction to ensure E-stability. ${ }^{3}$ When all agents use the same PLM, the equilibrium constitutes a Rational Expectations Equilibrium (REE).

2 In the homogenous expectations case, $\phi=\left(a_{1}\right)$ for $P L M_{1}$ and $\phi=\left(\begin{array}{c}a_{2} \\ b_{2}\end{array}\right)$ for $P L M_{2}$.

3 This condition brings forward many doubts on the usefulness of this literature. It turns out that this model is stable for any $-1<b<1$ and any reasonable choice of $a$. This is just assuming that agents believe the process to be stationary which is not, by any means, a very strong assumption. 
It turns out that the REE's are E-stable for only some values of $\beta_{0}$ and $\beta_{1}$. These E-stability conditions are expressed graphically in figure 1 which will be useful to compare these conditions with the E-stability conditions for heterogeneous expectations.

FIGURE 1. E-stability Conditions for Homogenous Expectations

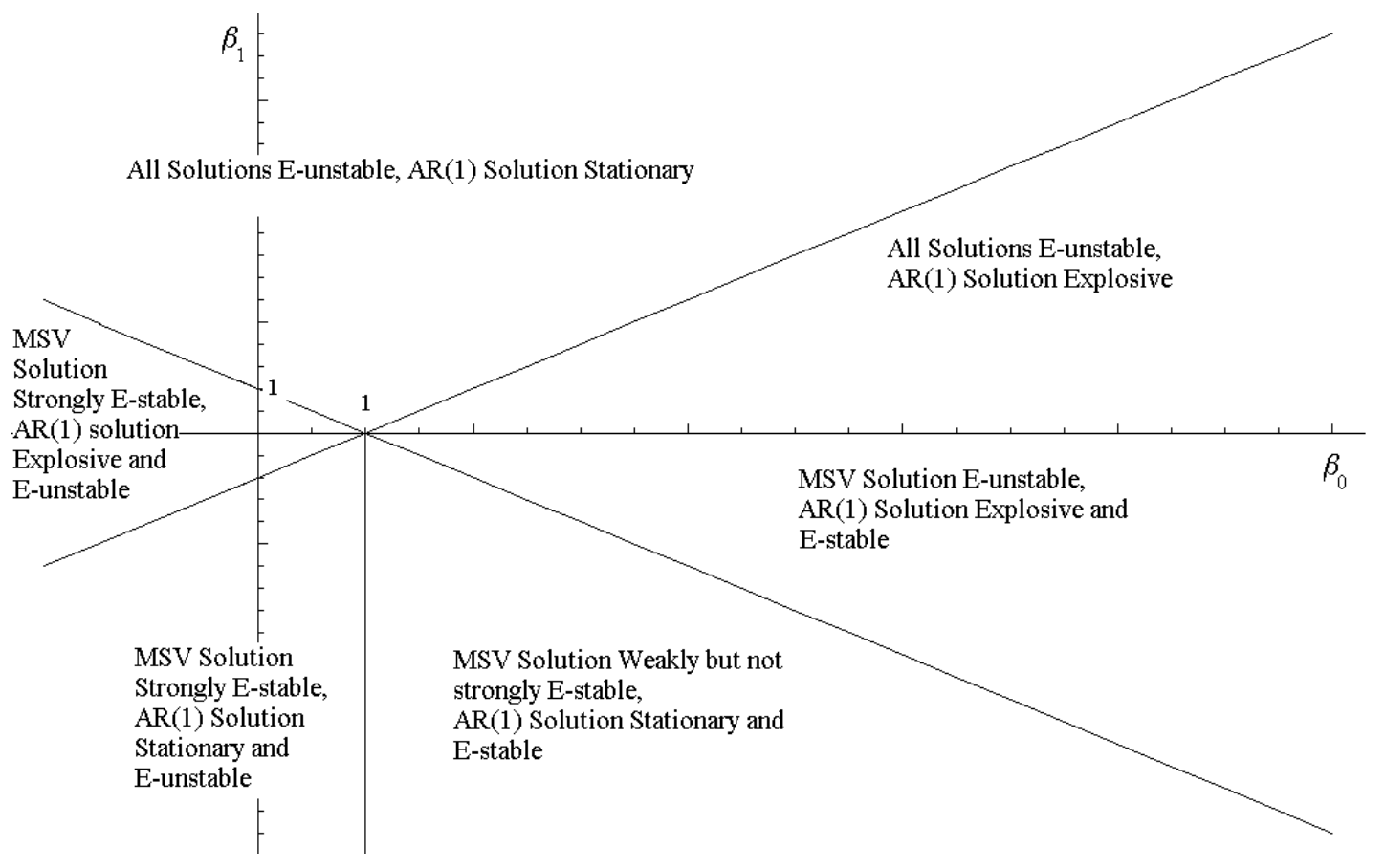

If agents use $P L M_{1}$, it turns out that the condition for E-stability is

$$
\beta_{0}+\beta_{1}<1
$$

This is known as the weak E-stability condition for the MSV solution. One can also find the strong E-stability conditions for the MSV solution which are the E-stability conditions for the MSV solution where all agents use $P L M_{2}$. The strong E-stability conditions are

$$
\beta_{0}<1 \text { and } \beta_{0}+\beta_{1}<1 \text {. }
$$

The E-stability conditions for the $\mathrm{AR}(1)$ solution for $P L M_{2}$ are

$$
\beta_{0}>1 \text { and } \beta_{1}<0 \text {. }
$$

It is not possible for the MSV solution to be strongly E-stable and the AR(1) solution to be weakly E-stable which will be an important fact in the case of heterogenous expectations. 


\subsection{The Model with Heterogenous Expectations}

For heterogeneous expectations, assume that there are two types of agents. One type uses the MSV PLM, or $P L M_{1}$, while the other uses the AR(1) PLM, or $P L M_{2}$. These agents may differ because the first type of agents are not willing to calculate the values for both $a$ and $b$, so they decide to just find the average of $y$. Assume that there is a fixed proportion of agents who use $P L M_{1}$ and agents who use $P L M_{2}$. This assumption will later be relaxed in the paper. Let $\mu$ be referred to as the proportion of agents that use the MSV PLM. Therefore, a proportion of $\mu$ agents form expectations using $P L M_{1}$ :

$$
P L M_{1}: y_{t}=a_{1}+v_{t}
$$

and a proportion of $(1-\mu)$ agents form expectations using $P L M_{2}$ :

$$
P L M_{2}: y_{t}=a_{2}+b_{2} y_{t-1}+v_{t}
$$

The actual law of motion (ALM) is formed from the averaged PLM of the agents, or $P L M_{A}$ :

$$
P L M_{A}: y_{t}=\mu a_{1}+(1-\mu) a_{2}+(1-\mu) b_{2} y_{t-1}+v_{t}
$$

\section{The ALM}

By combining equations 1 and 13, I obtain:

$$
\begin{aligned}
y_{t}= & \alpha+\mu a_{1}\left(\beta_{0}+\beta_{1}+\beta_{1}(1-\mu) b_{2}\right)+(1-\mu) a_{2}\left(\beta_{0}+\beta_{1}+\beta_{1}(1-\mu) b_{2}\right) \\
& +\left[(1-\mu) b_{2}\left(\beta_{0}+(1-\mu) \beta_{1} b_{2}\right)\right] y_{t-1}+v_{t}
\end{aligned}
$$

This equation describes the actual stochastic process followed by $y_{t}$ given the PLM's of the two types of agents. For each type, I now compute, for this $y_{t}$ process, the actual PLM parameters that best fit the $y_{t}$ process (in the mean square sense). I will call these implied parameters the ALM. For type 2 agents, the ALM parameters are simply the intercept and slope parameters of the implied $y_{t}$ process. Because, for $\mu<1$, agents using $P L M_{1}$ underparameterize the $y_{t}$ stochastic process, I need to compute for them the parameters of the $y_{t}$ process projected onto the space of $P L M_{1}$. Since type 1 agents model the process as iid, this is simply given by the mean of the implied $y_{t}$ process. 
The mapping from the average PLM to the ALM is thus the following: ${ }^{4}$

$$
\begin{aligned}
& T\left(\begin{array}{l}
a_{1} \\
a_{2} \\
b_{2}
\end{array}\right) \\
= & \left(\begin{array}{c}
\alpha+\mu a_{1}\left(\beta_{0}+\beta_{1}+\beta_{1}(1-\mu) b_{2}\right)+(1-\mu) a_{2}\left(\beta_{0}+\beta_{1}+\beta_{1}(1-\mu) b_{2}\right) \\
(1-\mu) b_{2}\left(\beta_{0}+(1-\mu) \beta_{1} b_{2}\right)
\end{array}\right)
\end{aligned}
$$

where $T_{a 1}{ }^{5}$ is a "projected ALM"6 assuming that the model follows a stationary process $\left(-1<b_{2}<1\right)$. Note that the first component of the T-map is given by:

$$
T_{a 1}=\frac{T_{a 2}}{1-T_{b 2}}
$$

\section{The Mixed Expectations Equilibria}

Next, I solve for the Mixed Expectations Equilibrium (MEE), where are defined as the fixed points of the T-mapping. ${ }^{7}$ The agents that use $P L M_{2}$ have the correct form of the economy and therefore their solution is rational within the equilibrium. Those agents using $P L M_{1}$ underparameterize the economy; although their mean is correct, their forecast errors are correlated with $y_{t-1}$ and hence their expectations are not as precise as the agents using $P L M_{2}$. The learned solution for the agents using $P L M_{1}$ is optimal given their restricted class of PLM's. It is easily verified from the T-map that the MEE are:

$$
\begin{aligned}
a_{1} & =\frac{\alpha}{1-b_{2}-\mu \lambda-(1-\mu)\left(1-b_{2}\right) \lambda}, \\
a_{2} & =\frac{\left(1-b_{2}\right) \alpha}{1-b_{2}-\mu \lambda-(1-\mu)\left(1-b_{2}\right) \lambda}, \text { and } \\
b_{2} & =\frac{1-(1-\mu) \beta_{0}}{(1-\mu)^{2} \beta_{1}}
\end{aligned}
$$

\footnotetext{
4 For convenience, I call this the ALM, although for type 1 agents the T-map gives the projected ALM.

$5 T_{a 1}$ denotes the first component of the map.

6 This is called a "projected ALM" since these agents use a misperceived law of motion which does not have the same form as the ALM. Note that if the solution is in the MSV form, then these agents asymptotically do not have a misspecified learning rule.

7 Since the agents using $P L M_{1}$ underparameterize the economy, when the equilibrium is $\mathrm{AR}(1)$, the equilibrium found by these agents will be referred as the Restricted Perceptions Equilibrium.
} 
or

$$
\begin{aligned}
a_{1} & =\frac{\alpha}{1-\beta_{0}-\beta_{1}}, \\
a_{2} & =\frac{\alpha}{1-\beta_{0}-\beta_{1}}, \text { and } \\
b_{2} & =0
\end{aligned}
$$

where:

$$
\lambda=1+\beta_{1}+\mu \beta_{0} .
$$

As with homogenous expectations where all agents use $P L M_{2}$, there are two equilibria: the $\mathrm{AR}(1)$ solution 17 and the MSV solution 18. It is important to note that the MSV MEE is the same as the MSV REE under homogeneous expectations. The AR(1) MEE is a combination of the MSV REE and the AR(1) REE since both PLM's are being used. ${ }^{8}$

\section{E-Stability}

Next, I solve for the E-stability conditions ${ }^{9}$ which is done by forming an ordinary differential equation (ODE) of the following:

$$
\frac{d \phi}{d \tau}=T(\phi)-\phi
$$

For stability, the eigenvalues of the Jacobian matrix of this above expression must have negative real parts. ${ }^{10}$ Figure 2 graphically presents all the E-stability results found below.

\subsection{E-stability Results for the MSV MEE and AR(1) MEE}

The E-stability results of the MSV MEE and the AR(1) MEE are stated in the following proposition:

Proposition 1: E-stability conditions for the above linear stochastic model with heterogeneous expectations.

8 It can be easily check that the MEE for extreme values of $\mu$ correspond to the REE for the homogenous agents case.

$9 \quad$ E-stability is applicable only if the stability of the stochastic recursive algorithm for the model can be determined by the E-stability equation 19. This is demonstrated in the appendix.

10 See the appendix for the ODE and the eigenvalues of the Jacobian matrix. 
1. All MSV MEE in the set

$$
E S_{1}=\left\{\left(\beta_{0}, \beta_{1}\right) \mid \beta_{0}<\left(\frac{1}{1-\mu}\right), \beta_{0}+\beta_{1}<1\right\}
$$

are E-stable. All MSV MEE outside of this set are E-unstable.

2. All AR(1) MEE in the set

$$
E S_{2}=\left\{\begin{array}{c}
\left(\beta_{0}, \beta_{1}\right) \mid \frac{1}{1-\mu}<\beta_{0}<\frac{1}{1-\mu}-(1-\mu) \beta_{1}, \\
\beta_{1}<0
\end{array}\right\}
$$

are E-stable. All AR(1) MEE outside of this set are E-unstable. ${ }^{11}$

\subsection{Graphical Representation of the E-stability Conditions}

Figure 2 presents the graph for the E-stability conditions under heterogeneous expectations. 1 represents $\beta_{0}=1,2$ represents $\beta_{0}+\beta_{1}=1,3$ represents $\beta_{0}=\frac{1}{1-\mu}$, and 4 represents $\beta_{1}=\frac{1-\beta_{0}(1-\mu)}{(1-\mu)^{2}}$. The original homogenous MSV strong E-stability condition was represented by area $\mathrm{A}$ in figure 2. Heterogenous expectations now adds area $\mathrm{B}$ to $\mathrm{A}$ as the area of MSV E-stability. Area B represents an area that was weakly MSV E-stable but not strongly MSV E-stable for homogenous expectations. This area now represents part of the MSV E-stable area since some of the agents use the MSV PLM. The E-stability conditions for the MSV MEE are now dependent upon $\mu$, the proportion of agents using the MSV predictor.

11 In this paper, I do not examine stability properties when some agents use higher order PLM's. 
FIGURE 2. E-stability Conditions for Heterogeneous Expectations

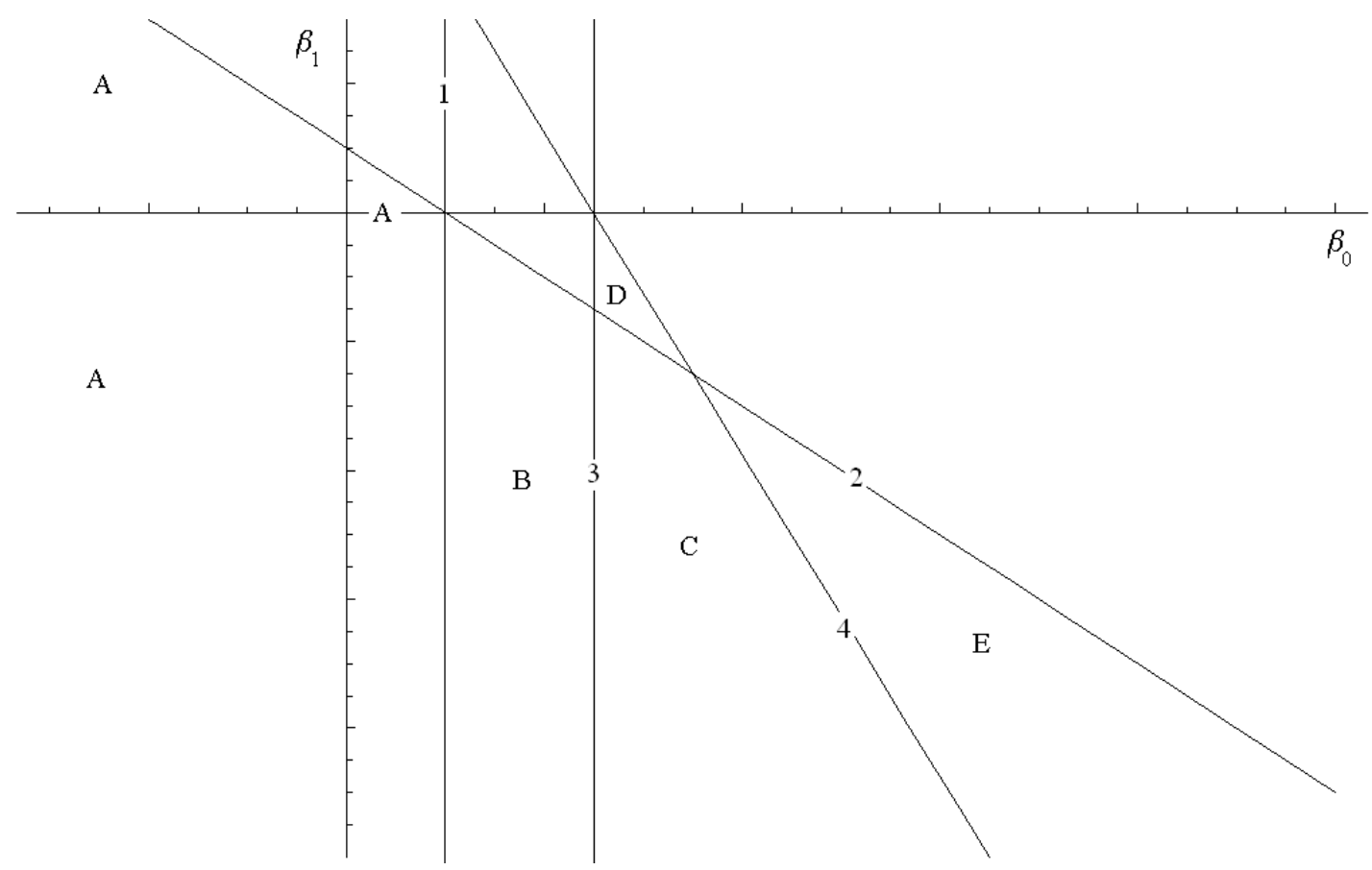

These E-stability conditions reflect a combination of the strong E-stability and weak E-stability conditions from the MSV REE with homogenous expectations. The restriction on $\beta_{0}$ decreases as the proportion of agents using $P L M_{1}$ increases. Since both the weak and strong E-stability results for homogeneous expectations have the inequality $\beta_{0}+\beta_{1}<1$ in common, intuition tells us that $\mu$ should not be included in this stability condition. These results produce the following corollary: Corollary 1: E-Stability conditions for the MSV MEE from the above model become less stringent, with respect to $\beta_{0}$, as the proportion of agents that use the MSV predictor increases.

Less stringent means that as $\mu$ increases, the maximum value for $\beta_{0}$ necessary for MSV E-stability increases. Note that as $\beta_{0}$ is allowed to increase, the model requires a smaller value of $\beta_{1}$ for MSV E-stability. This is due to the condition: $\beta_{0}+\beta_{1}<1$ necessary for MSV E-stability.

Now consider the AR(1) E-stability conditions represented by regions C and D in figure 2. Region D represents an area that was non-stationary with homogenous expectations, but it is now stationary and E-stable for a given $\mu$. E-stability conditions rule out area E which was AR(1) E-stable and stationary with homogenous expectations. Area E now represents the MEE that are non-stationary that would be stationary if all agents used the AR(1) PLM. 
The E-stability conditions for the AR(1) MEE are a hybrid of both the E-stability conditions for the MSV REE and the AR(1) REE under homogeneous expectations. The stability condition

$$
\beta_{0}>\frac{1}{1-\mu}
$$

is a form of the E-stability condition for the $\operatorname{AR}(1)$ solution in the homogenous case where $\beta_{0}>1$. The stability condition

$$
0>\left(\frac{(1-\mu)^{2} \beta_{1}\left(1-\beta_{0}-\beta_{1}\right)}{1-(1-\mu) \beta_{0}-(1-\mu)^{2} \beta_{1}}\right)
$$

is a combination of the two conditions for $\operatorname{AR}(1)$ E-stability

$$
\begin{aligned}
& \beta_{0}>1 \\
& \beta_{1}<0,
\end{aligned}
$$

the strong E-stability conditions for the MSV solution

$$
\begin{array}{r}
\beta_{0}+\beta_{1}<1 \\
\beta_{0}<1,
\end{array}
$$

all divided by the condition for stationarity

$$
\beta_{1}<\frac{1}{(1-\mu)^{2}}-\frac{1}{(1-\mu)} \beta_{0} .
$$

With these two stability conditions for the model, I will examine how the agents who misspecify the model affect the stability of the equilibrium. In other words, what happens to the stability of the equilibria when the value of $\mu$ increases?

Corollary 2: For any given $\left(\beta_{0}, \beta_{1}\right)$ that is AR(1) E-stable for some $\mu_{0}$, there exists a $\mu_{1}>\mu_{0}$ such that the AR(1) MEE is E-unstable.

As more agents use $P L M_{1}$, the E-stable MEE will eventually not be the solution corresponding to the "minority" of agents using $P L M_{2}$.

\section{The Role of Heterogeneity}

Corollary 2 shows that if the level of heterogeneity, $\mu$, is arbitrarily changed, then a MEE may transform from being E-stable to E-unstable. I now consider in detail the implications of varying $\mu$. For this exercise to be well defined, I need to require that every AR(1) MEE be stochastically stationary. This is because If the MEE is non-stationary, then the agents using $P L M_{1}$ will be 
using an obviously misspecified learning rule. I will therefore only consider models in which all AR(1) solutions are stationary for all $\mu \in\left[0,1-\frac{1}{\beta_{0}}\right){ }^{12}$

\subsection{Stationarity for Heterogenous Expectations}

Figure 3 shows the values of $b_{2}$ for the AR(1) MEE as $\mu$ changes. Some of the equilibrium values from the $\operatorname{AR}(1)$ solution (17) may be non-stationary for some values of $\mu, \beta_{0}$, and $\beta_{1}$. Therefore, I now restrict $\beta_{0}$ and $\beta_{1}$ such that the $\operatorname{AR}(1) \mathrm{MEE}$ is stationary for any $\mu \in\left[0,1-\beta_{0}^{-1}\right)$.

FIGURE 3. The value of $b_{2}$ of the AR(1) Solution as $\mu$ Varies.

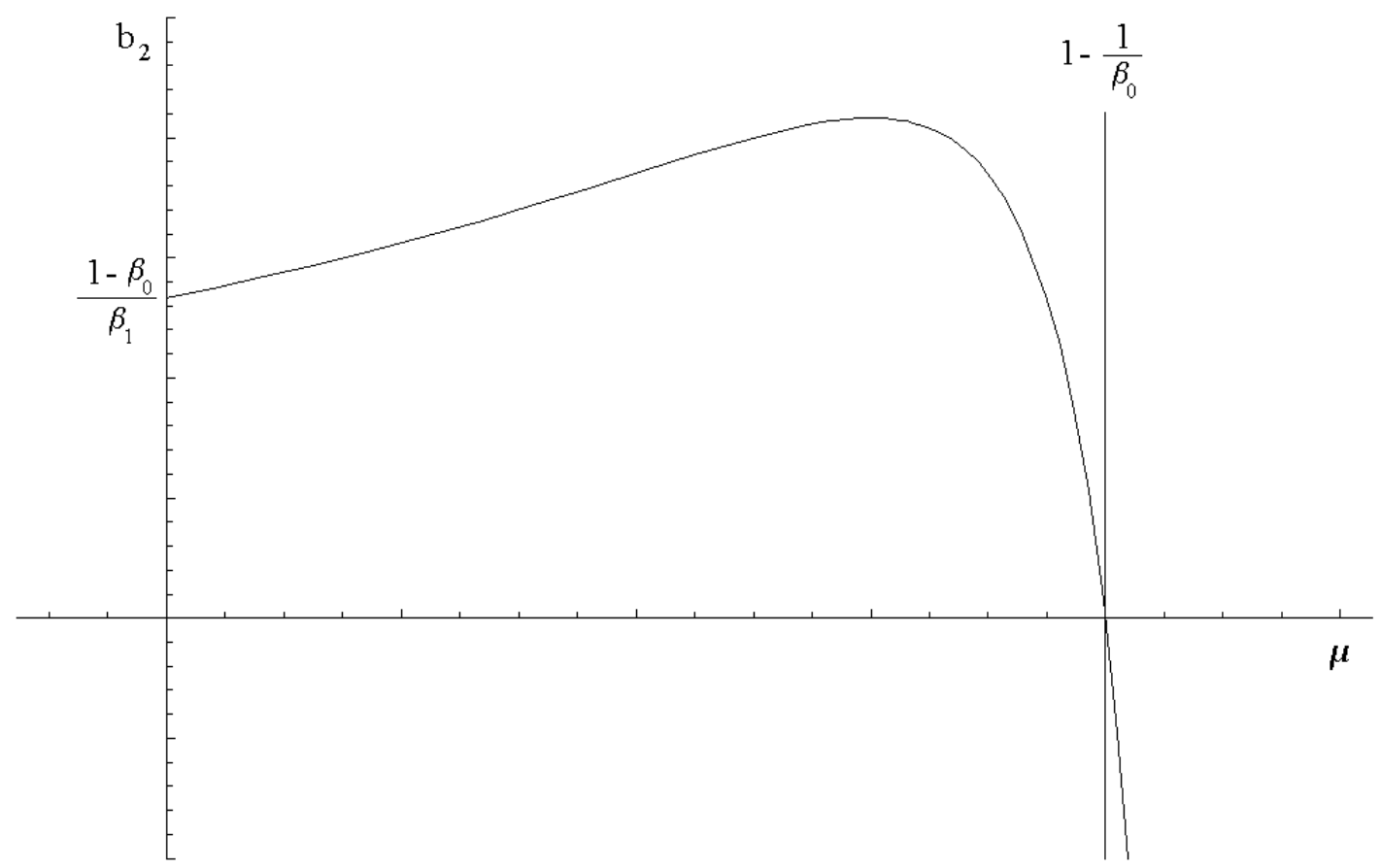

To restrict $-1<b_{2}<1$, it must be that

$$
\max b_{2}<1
$$

and

$$
\min b_{2}>-1
$$

12 For any $\mu>1-\beta_{0}^{-1}$, the $\operatorname{AR}(1)$ MEE is not E-stable, while the MSV solution is always stationary, so the issue of stationarity does not arise for $\mu>1-\frac{1}{\beta_{0}}$. 
In the appendix, it is shown that for $b_{2}<1$, it must be that

$$
\beta_{1}<-\frac{1}{4} \beta_{0}^{2} .
$$

Assume that the values of $\beta_{0}$ and $\beta_{1}$ always satisfy the above condition. This rules out the shaded areas represented in figure 4 . A restriction for $\beta_{0}$ and $\beta_{1}$ will not be necessary for $-1<b_{2}$ since the AR(1) MEE is not E-stable for

$$
\mu>1-\frac{1}{\beta_{0}} .
$$

No additional assumption for the MSV MEE is necessary because there is no problem of stationarity for this solution.

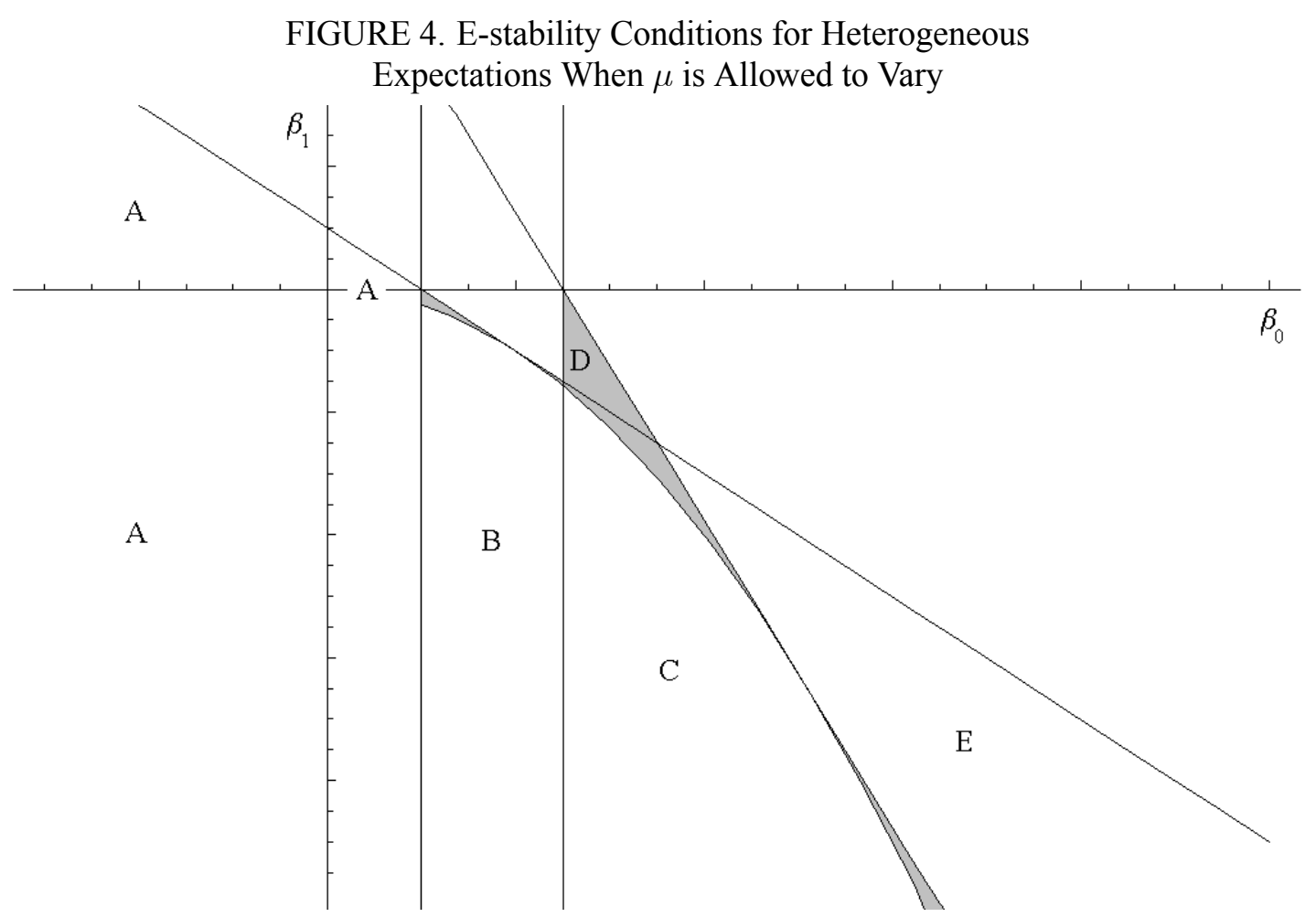

\subsection{Switching}

For parameters restricted to this region, at least one of the two MEE's is E-stable for all $\mu \in[0,1]$. However, for part of the region, the equilibria exchange E-stability when the proportion of agents using the MSV predictor, $\mu$, is changed. 
Definition 2: Switching is said to occur at $\left(\beta_{0}, \beta_{1}\right)$ if $(i)$ for all $\mu \in[0,1]$, exactly one MEE is E-stable and (ii) there exists $\mu^{c} \in(0,1)$ such that the AR(1) MEE is E-stable for $\mu<\mu^{c}$ and the MSV MEE is E-stable for $\mu>\mu^{c}$.

Recall that $\mu=1-\beta_{0}^{-1}$ is the point where the solution changes from being MSV E-stable to AR(1) E-stable. As the value of $\mu$ increases, the level curve, $\beta_{0}=\frac{1}{1-\mu}$, shifts to the right. With a large enough increase in $\mu$, the model will transform from being AR(1) E-stable to being MSV E-stable. The following proposition examines the limits of this level curve to establish the region of E-stability switching.

Proposition 2: Switching occurs within the set

$$
S=\left\{\left(\beta_{0}, \beta_{1}\right) \mid \beta_{0}>1, \beta_{1}<-\frac{1}{4} \beta_{0}^{2}\right\} .
$$

This region described above is the same region of weak E-stability of the AR(1) REE and the MSV REE in the homogenous expectations case with the restricted stationarity conditions. This area is represented as areas B and C in figure 4. Adding more agents that use a particular PLM to the model makes the solution corresponding to that particular PLM more likely to be E-stable.

\subsection{The Mean Squared Error and Switching}

Next, consider the mean squared error (MSE) for each estimator under each equilibrium. The MSE is calculated in the appendix and is the following for the AR(1) MEE:

$$
\begin{aligned}
& M S E_{1}=\frac{(1-\mu)^{4} \sigma^{2} \beta_{1}^{2}}{(1-\mu)^{4} \beta_{1}^{2}-\left(1-(1-\mu) \beta_{0}\right)^{2}} \geq \sigma^{2} \\
& M S E_{2}=\sigma^{2}
\end{aligned}
$$

where the subscript denotes the number of the PLM used as a predictor. The MSE for $P L M_{1}$ is never less than the MSE for $P L M_{2}$, for the AR(1) MEE, since $P L M_{1}$ is the inefficient estimator of the solution. The MSE for the MSV MEE is the following:

$$
M S E_{1}=M S E_{2}=\sigma^{2}
$$

In this case, each predictor has the same asymptotic quality of prediction. This brings forward the question "why does E-stability switch from the AR(1) solution to the MSV solution for different values of $\mu$ ?" The answer to this question is the following proposition: 
Corollary 3: Exactly one solution can be E-stable for any $\left(\beta_{0}, \beta_{1}\right)$ for the heterogeneous expectations case. If $\beta_{1}<-\frac{1}{4} \beta_{0}^{2}$ and $\beta_{0}>1$, the AR(1) MEE and MSV MEE exchange E-stability when the MSE of the first predictor (with an AR(1) solution) is minimized with respect to $\mu$.

It turns out that the minimum of $M S E_{1}$ is $\sigma^{2}$. The two equilibria, at this value of $\mu$, are equal to the MSV MEE where $b_{2}=0$. Therefore, this is the point where both predictors are efficient, or $M S E_{1}=M S E_{2}=\sigma^{2}$. When the MSE's of the solutions are equal, there is an exchange of stability between the two solutions. As more agents use a specific predictor, the solution that is E-stable is the solution corresponding to that predictor. When $\mu$ is larger than its switching point value, the MSV predictor is no longer asymptotically inefficient, since the AR(1) solution changes from a sink to a source. Hence, here the MSV solution is E-stable while the AR(1) solution is not. When $\mu$ is smaller than the above value, the inefficiency of the MSV predictor and the fact that more of the agents are using $P L M_{2}$ makes the AR(1) solution E-stable and the MSV solution E-unstable.

\subsection{Agent Dynamic E-stability}

Finally, I examine the region where switching never occurs, but one MEE is always E-stable for all $\mu \in[0,1]$. This will be referred to as Agent Dynamic E-stability.

Definition 3: A MEE is Agent Dynamic E-stable (ADE) if it is E-stable for all $\mu \in[0,1]$.

When a MEE is Agent Dynamic E-stable, no change in agents beliefs can change the stability of either MEE. E-stability of this solution does not depend upon the proportion of agents using $P L M_{1}$, so switching will never occur. The region of ADE turns out to be the same region as another important area in the homogenous agent problem.

Proposition 3: The region of $A D E$ in the heterogenous agent case is equal to the region of strong E-stability for the MSV solution in the homogenous agents case.

The region of $\mathrm{ADE}$ is represented by area $\mathrm{A}$ in figure 4. The above proposition gives information on the nature of E-stability of the MEE's in the ADE region. The reason the AR(1) MEE is never E-stable here is that for homogenous expectations, it is not E-stable here. This intuition produces the following corollary:

Corollary 4: For the model presented above, the only MEE that is ADE is the MSV solution. 


\section{Conclusion}

This paper has studied several asymptotic convergence properties of the above model under heterogenous expectations. The E-stability conditions were observed for the model for both the AR(1) and Minimum State Variable (MSV) solutions. I then observed the stability conditions of the model under learning when heterogeneity was allowed to vary. For this, I examined both the mean squared error for using each predictor and also the restrictions for the AR(1) solution to always be stationary for all E-stable solutions.

The AR(1) solution is affected by heterogeneous expectations, but the MSV solution does not change with heterogeneous expectations. E-stability of the mixed expectations equilibrium (MEE) for both the MSV and AR(1) solutions depends upon the proportion of agents who believe that the economy follows an independent and identically distributed (i.i.d.) process. If this proportion of heterogeneity were different, some solutions that are E-stable for the current proportion of heterogeneity become E-unstable solutions.

Finally, I ask "what are the stability conditions if we can observe any level of heterogeneity?" It turns out that for the agents using the i.i.d. predictor to learn a solution, the equilibrium must be stationary for any level of heterogeneity. I find the conditions for stationarity for all previously E-stable equilibria. From this set of possible equilibria, two phenomena may occur. First, for an exogenously changing level of heterogeneity, it is possible that one initially E-stable equilibrium may become E-unstable while the other equilibrium becomes E-stable at this point of change. There is an exchange of stability between the two equilibria at the level of heterogeneity where the mean squared error of the MSV predictor is minimized. Second, in another region of the parameter space, the MSV equilibrium may be E-stable for all possible levels of heterogeneity.

Heterogeneity adds another kink to the armor of the rational expectations hypothesis. For agents to possess RE, they must know the structure of the equilibrium as well as the expectations made by all other agents in the economy. Because of this additional strong assumption, the bounded rationality literature becomes even more attractive as an alternative paradigm for modelling expectations. 


\section{Appendix}

\subsection{Proofs}

Proof of Proposition 1 An equilibrium is E-stable if the E-stability differential equation

$$
\frac{d \phi}{d \tau}=T(\phi)-\phi
$$

is stable. This means the eigenvalues of the Jacobian matrix of this ODE must all have negative real parts.

1. When we insert the value of $b_{2}=0$ into the eigenvalues of the Jacobian matrix, we get the following eigenvalues: ${ }^{13}$

$$
-1,\left(\frac{1}{1-\mu}\right)-\beta_{0}, \beta_{0}+\beta_{1}-1
$$

All three of these values are negative when:

$$
\begin{aligned}
\beta_{0} & <\left(\frac{1}{1-\mu}\right) \\
\beta_{0}+\beta_{1} & <1
\end{aligned}
$$

When any of these conditions are not met, at least one of the eigenvalues has positive real parts making the ODE unstable. Therefore, for the MSV MEE to be E-stable, the parameters must be contained in the above mentioned set.

2. When we insert the value of $b_{2}=\frac{1-(1-\mu) \beta_{0}}{(1-\mu)^{2} \beta_{1}}$ into the eigenvalues of the Jacobian matrix, we get the following eigenvalues: ${ }^{14}$

$$
-1,1-(1-\mu) \beta_{0},\left(\frac{(1-\mu)^{2} \beta_{1}\left(1-\beta_{0}-\beta_{1}\right)}{1-(1-\mu) \beta_{0}-(1-\mu)^{2} \beta_{1}}\right)
$$

13 It can be easily shown that for $\mu=1$, the eigenvalues are $\left\{-1,-1,-1+\beta_{0}+\beta_{1}\right\}$. This turns out to be the MSV weak E-stability condition for the homogenous agents case.

14 For $\mu=0$, it turns out the eigenvalues are: $\left\{-1,-1+\beta_{0}+\left(1+b_{2}\right) \beta_{1}, \beta_{0}+2 \beta_{1} b_{2}-1\right\}$. This is the result for $\mathrm{AR}(1)$ weak E-stability in the homogenous agents case. 
All three of these values are negative when:

$$
\begin{aligned}
\beta_{0} & >\left(\frac{1}{1-\mu}\right) \\
0 & >\left(\frac{(1-\mu)^{2} \beta_{1}\left(1-\beta_{0}-\beta_{1}\right)}{1-(1-\mu) \beta_{0}-(1-\mu)^{2} \beta_{1}}\right)
\end{aligned}
$$

Consider inequality 33 for E-stability. We can re-write this equation as

$$
0>\left(\frac{\beta_{1}\left(1-\beta_{0}-\beta_{1}\right)}{1-(1-\mu) \beta_{0}-(1-\mu)^{2} \beta_{1}}\right) .
$$

Let us consider the case where $\beta_{1}<0$. Since there are some agents that use PLM $M_{1}$, the model must be stationary. Therefore,

This can be further written as

$$
b_{2}=\frac{1-(1-\mu) \beta_{0}}{(1-\mu)^{2} \beta_{1}}<1 .
$$

$$
1-(1-\mu) \beta_{0}-(1-\mu)^{2} \beta_{1}>0 .
$$

Therefore, for inequality 33 to hold, it must be that

$$
\beta_{0}+\beta_{1}<1 .
$$

The stationarity condition is more restrictive than this, so it turns out that the stationarity condition:

$$
\beta_{0}<\frac{1}{1-\mu}-(1-\mu) \beta_{1}
$$

will be one of the conditions for E-stability in this model.

Now consider the case where $\beta_{1}>0$. For stationarity, it turns out that we need:

$$
1-(1-\mu) \beta_{0}-(1-\mu)^{2} \beta_{1}<0 .
$$

This means that it must be that

$$
1-\beta_{0}-\beta_{1}>0
$$

or

$$
\beta_{0}<1-\beta_{1} \text {. }
$$

Our first E-stability condition

$$
\beta_{0}>\frac{1}{1-\mu}
$$

will not be met in this case. Therefore, for the AR(1) MEE to be E-stable, the following must hold:

$$
\begin{aligned}
\frac{1}{1-\mu} & <\beta_{0}<\frac{1}{1-\mu}-(1-\mu) \beta_{1} \\
\beta_{1} & <0 .
\end{aligned}
$$


When one of these above stated conditions are not met, then either one of the eigenvalues of the Jacobian matrix are positive making the ODE unstable or the model is not stationary. In this case, the model is E-unstable. Therefore, for the AR(1) MEE to be E-stable, the parameters must be contained within the above mentioned set.

Proof of Corollary 1 The derivative from level curve 30 is

$$
\frac{\partial \beta_{0}}{\partial \mu}=(1-\mu)^{-2}>0
$$

Notice that $\beta_{0}>(1-\mu)^{-1}$ for the AR(1) MEE and that $\beta_{0}<(1-\mu)^{-1}$ for the MSV MEE. The critical value of $\beta_{0}$ for MSV E-stability increases as $\mu$ increases. This increase in $\mu$ makes MSV E-stability less stringent while AR(1) E-stability becomes more stringent.

Proof of Corollary 2 If $\left(\beta_{0}, \beta_{1}\right)$ is AR(1) E-stable, then $\beta_{0}>\left(1-\mu_{0}\right)^{-1}$ and condition 33 holds. Since $\mu_{0} \in[0,1)$, then there exists a $\mu_{1}>\mu_{0}$. It follows that

$$
\frac{1}{1-\mu_{0}}<\frac{1}{1-\mu_{1}} \in(1, \infty) \text {. }
$$

Choose $\mu_{1}$ such that $\left(1-\mu_{1}\right)^{-1}>\beta_{0}$. Now the model is no longer AR(1) E-stable.

Proof of Proposition 2 For switching to occur we must be in a region where both types of E-stability exist for some $\mu \in[0,1]$. For the AR(1) MEE to be potentially E-stable for all $\mu \in[0,1)$, it must be that the solution will be stationary for all $\mu \in[0,1)$. Therefore, it must be that $\beta_{1}<-\frac{1}{4} \beta_{0}^{2}$ as discussed previously. This is the only possible region where the switching will occur. The second necessary condition for this E-stability switching is that the level curve $\beta_{0}^{c}=(1-\mu)^{-1}$ can cross the point of $\left(\beta_{0}, \beta_{1}\right)$. Since $\mu \in[0,1]$, then $\beta_{0}^{c} \in(1, \infty)$. From this, it must be that $\beta_{0}>1$ for switching to occur. Therefore, the bounds of the E-stability switching are the following: $\beta_{0}>1$ and $\beta_{1}<-\frac{1}{4} \beta_{0}^{2}$.

Proof of Proposition 3 In the homogenous expectations case, both the AR(1) and MSV solutions are weakly E-stable in the set $S=\left\{\left(\beta_{0}, \beta_{1}\right) \mid \beta_{0}>1, \beta_{1}<-\frac{1}{4} \beta_{0}^{2}\right\}$. The union of the two sets describing E-stability of the two solutions, in the heterogeneous expectations case, is: $E S_{1} \cup E S_{2}=\emptyset$. Therefore, both of the solutions can not be E-stable for any $\left(\beta_{0}, \beta_{1}\right)$. In the heterogenous expectations case, if $\beta_{1}<-\frac{1}{4} \beta_{0}^{2}$, then inequality 33 always holds. Since $\beta_{0}>1$, there is some $\mu$ such that the AR(1) solution is E-stable. Therefore, if $\beta_{1}<-\frac{1}{4} \beta_{0}^{2}$, then the level curve distinguishing the difference between MSV and AR(1) E-stability is $\beta_{0}=(1-\mu)^{-1}$. The 
derivative of $M S E_{1}$ is zero if:

$$
\begin{aligned}
& 2(1-\mu)^{3}\left(2-(1-\mu) \beta_{0}\left(3-(1-\mu) \beta_{0}\right)\right) \sigma^{2} \beta_{1}^{2}=0 \\
& \quad \Rightarrow \quad 2\left(2-(1-\mu) \beta_{0}\left(3-(1-\mu) \beta_{0}\right)\right) \sigma^{2} \beta_{1}=0 \\
& \quad \Rightarrow \quad\left(2-(1-\mu) \beta_{0}\left(3-(1-\mu) \beta_{0}\right)\right)=0 \\
& \Rightarrow \mu=1-\frac{2}{\beta_{0}} \text { and } \mu=1-\frac{1}{\beta_{0}}
\end{aligned}
$$

The second solution is exactly the switching point of E-stability of the above model as long as $\beta_{1}<-\frac{1}{4} \beta_{0}^{2}$. Checking the SOC, we find that

$$
\mu=1-\frac{2}{\beta_{0}}
$$

is a maximum for the $M S E_{1}$, while

$$
\mu=1-\frac{1}{\beta_{0}}
$$

is a minimum for the $M S E_{1}$.

Proof of Proposition 3 From the homogenous agent case, the region of strong E-stability in the above model is $A=\left\{\left(\beta_{0}, \beta_{1}\right) \mid \beta_{0}<1, \beta_{0}+\beta_{1}<1\right\}$. In order for no E-stability switching to occur, we must be outside of the set $S$ mentioned above. Therefore, it must be that $\beta_{0}<1$. Moreover, to have ADE we must always be in a region where the MEE is always E-stable. This only occurs when we have $\beta_{0}+\beta_{1}<1$. This is the region of strong E-stability for the MSV solution in the homogenous agents case.

Proof of Corollary 4 The region of strong ADE is contained entirely in the region of MSV E-stable MEE's and outside of the region of AR(1) E-stable MEE's.

\subsection{The Stochastic Recursive Algorithm}

To show that E-stability is applicable for this model, we must show that stability of the stochastic recursive algorithm can be determined by exactly the E-stability equation:

$$
\frac{d \phi}{d \tau}=T(\phi)-\phi .
$$


The model with the two learning rules presented above can be described using recursive least squares. The model in RLS form is:

$$
\begin{aligned}
\phi_{t} & =\phi_{t-1}+t^{-1}\left(S_{t-1}^{-1} z_{t-1}\left(\begin{array}{c}
\left.y_{t}-a_{1, t-1}-z_{t-1}^{\prime}\left(\begin{array}{c}
a_{2, t-1} \\
b_{2, t-1}
\end{array}\right)\right)
\end{array}\right)\right. \\
S_{t} & =S_{t-1}+t^{-1}\left(z_{t} z_{t}^{\prime}-S_{t-1}\right)+t^{-2}\left(\frac{-t}{t+1}\right)\left(z_{t} z_{t}^{\prime}-S_{t-1}\right)
\end{aligned}
$$

where

$$
\begin{aligned}
\phi_{t-1}^{\prime} & =\left(a_{1, t-1}, a_{2, t-1}, b_{t-1}\right) \\
z_{t-1}^{\prime} & =\left(1, y_{t-1}\right) .
\end{aligned}
$$

Consider a stochastic recursive algorithm of the form

$$
\theta_{t}=\theta_{t-1}+\gamma_{t} Q\left(t, \theta_{t-1}, X_{t}\right)
$$

where $\theta_{t}$ is a vector of parameter estimates, $X_{t}$ is the state vector, and $\gamma_{t}$ is a deterministic sequence of gains. $Q$ describes the way in which the estimate $\theta_{t-1}$ is updated every period from the previous period's observations. The system 41-42 is implicitly in the standard form of the SRA $^{15}$ where:

$$
\begin{aligned}
\theta & =\operatorname{vec}\left(\phi_{t} S_{t}\right) \\
X_{t} & =\left(\begin{array}{c}
1 \\
y_{t} \\
y_{t-1} \\
v_{t}
\end{array}\right) \\
\gamma_{\tau} & =t^{-1} .
\end{aligned}
$$

The components of Q are the following:

$$
\begin{aligned}
Q_{\phi}\left(t, \theta_{t-1}, X_{t}\right) & =\left(\begin{array}{c}
z_{t-1}^{\prime} T_{2}\left(\phi_{t-1}\right)-a_{1, t-1}+v_{t} \\
S_{t-1}^{-1} z_{t-1}\left(z_{t-1}^{\prime}\left(T_{2}\left(\phi_{t-1}\right)-\phi_{2, t-1}\right)+v_{t}\right)
\end{array}\right) \\
Q_{S}\left(t, \theta_{t-1}, X_{t}\right) & =\operatorname{vec}\left(\left(z_{t} z_{t}^{\prime}-S_{t-1}\right)+t^{-1}\left(\frac{-t}{t+1}\right)\left(z_{t} z_{t}^{\prime}-S_{t-1}\right)\right)
\end{aligned}
$$

15 See Evans and Honkapohja (2001) for details of the stochastic recursive algorithm. See pp. 34-36, Chapter 6 for technical details, and Chapter 8 for details of the above model. 
where

$$
\begin{aligned}
Q & =\left(\begin{array}{c}
Q_{\phi}\left(t, \theta_{t-1}, X_{t}\right) \\
Q_{S}\left(t, \theta_{t-1}, X_{t}\right)
\end{array}\right) \\
T_{2}\left(\phi_{t-1}\right) & =\left(\begin{array}{c}
T_{a 2}\left(\phi_{t-1}\right) \\
T_{b 2}\left(\phi_{t-1}\right)
\end{array}\right) \\
\phi_{2, t-1} & =\left(\begin{array}{c}
a_{2, t-1} \\
b_{2, t-1}
\end{array}\right) .
\end{aligned}
$$

We have now set up the T-mapping such that $T_{1}$ and $T_{2}$ correspond with the PLM's of the two types of agents respectively. Notice that the ALM for the economy is governed entirely by the T-mapping of the agents with the correctly specified model.

Next, we can determine convergence of the SRA using stochastic approximation. Marcet and Sarget (1989) have shown how this technique presented in Ljung (1977) can be useful in the analysis of adaptive learning. The stochastic approximation approach associates an ordinary differential equation (ODE) with the SRA:

$$
\frac{d \theta}{d \tau}=h(\theta(\tau))
$$

where $h(\theta)$ is

$$
h(\theta)=\lim _{t \rightarrow \infty} E Q\left(t, \theta, X_{t}\right) .
$$

Following Evans and Honkapohja (2001), we get the following:

$$
\begin{aligned}
& h_{\phi}(\phi, S)=\lim _{t \rightarrow \infty} E\left(\begin{array}{c}
z_{t-1}^{\prime} T_{2}\left(\phi_{t-1}\right)-a_{1, t-1}+v_{t} \\
S_{t-1}^{-1} z_{t-1}\left(z_{t-1}^{\prime}\left(T_{2}\left(\phi_{t-1}\right)-\phi_{2, t-1}\right)+v_{t}\right)
\end{array}\right) \\
& h_{S}(\phi, S)=\lim _{t \rightarrow \infty} E\left(\left(z_{t} z_{t}^{\prime}-S_{t-1}\right)+t^{-1}\left(\frac{-t}{t+1}\right)\left(z_{t} z_{t}^{\prime}-S_{t-1}\right)\right)
\end{aligned}
$$

where

$$
h(\theta)=\left(\begin{array}{c}
h_{\phi}(\phi, S) \\
h_{S}(\phi, S)
\end{array}\right)
$$

If we assume $y_{t}$ follows a stationary process, then Equation 47 becomes the following:

$$
h_{\phi}(\phi, S)=\left(\begin{array}{c}
E\left(y_{t}\right)-a_{1, t-1} \\
S_{t-1}^{-1} E z_{t-1} z_{t-1}^{\prime}\left(T_{2}\left(\phi_{t-1}\right)-\phi_{2, t-1}\right)
\end{array}\right)
$$

Since

$$
\begin{aligned}
& E z_{t} z_{t}^{\prime}=E z_{t-1} z_{t-1}^{\prime}=\left(\begin{array}{cc}
1 & E\left(y_{t}(\phi)\right) \\
E\left(y_{t}(\phi)\right) & E\left(y_{t}(\phi)^{2}\right)
\end{array}\right) \equiv M_{z}(\phi), \\
& E\left(y_{t}\right)=T_{1}\left(\phi_{t-1}\right),
\end{aligned}
$$

and $E z_{t-1} v_{t}=0$, we have the following:

$$
\begin{aligned}
h_{\phi}(\phi, S) & =\left(\begin{array}{c}
T_{1}(\phi)-a_{1} \\
S^{-1} M_{z}(\phi)\left(T_{2}(\phi)-\phi_{2}\right)
\end{array}\right) \\
h_{S}(\phi, S) & =M_{z}(\phi)-S .
\end{aligned}
$$


The associated ODE from the system of SRA's becomes:

$$
\begin{aligned}
\frac{d \phi}{d \tau} & =\left(\begin{array}{c}
T_{1}(\phi)-a_{1} \\
S^{-1} M_{z}(\phi)\left(T_{2}(\phi)-\phi_{2}\right)
\end{array}\right) \\
\frac{d S}{d \tau} & =M_{z}(\phi)-S .
\end{aligned}
$$

This system is now recursive and note that equation 53 is a globally stable system with $S \rightarrow M_{z}(\phi)$ from any starting point. From this fact, it follows that $S^{-1} M_{z}(\phi) \rightarrow I$ from any starting point provided that $\mathrm{S}$ is invertible along the path. This means that the stability of the system can be determined entirely by the following ODE:

$$
\frac{d \phi}{d \tau}=\left(\begin{array}{c}
T_{1}(\phi)-a_{1} \\
T_{2}(\phi)-\phi_{2}
\end{array}\right)=T(\phi)-\phi
$$

Therefore, we can find the stability of the system by finding the E-stability conditions.

\subsection{Stationarity of the AR(1) MEE}

Here we solve for the maximum value for $b_{2}$. Recall that

$$
b_{2}=\frac{1-(1-\mu) \beta_{0}}{(1-\mu)^{2} \beta_{1}} .
$$

Therefore,

$$
\frac{\partial b_{2}}{\partial \mu}=\frac{(1-\mu)^{2} \beta_{0} \beta_{1}+\left[1-(1-\mu) \beta_{0}\right] 2(1-\mu) \beta_{1}}{(1-\mu)^{4} \beta_{1}^{2}}
$$

so the maximum is where

$$
(1-\mu)^{2} \beta_{0} \beta_{1}+\left[1-(1-\mu) \beta_{0}\right] 2(1-\mu) \beta_{1}=0
$$

From this we get:

$$
\mu=1-\frac{2}{\beta_{0}}
$$

Therefore,

$$
\begin{aligned}
\max b_{2} & =\frac{1-\frac{2}{\beta_{0}} \beta_{0}}{\left(\frac{2}{\beta_{0}}\right)^{2} \beta_{1}} \\
& =\frac{-\beta_{0}^{2}}{4 \beta_{1}} .
\end{aligned}
$$

\subsection{The ODE and the eigenvalues of the Jacobian matrix}

From the T-mapping we can get the following ODE: 


$$
\begin{aligned}
\frac{d \phi}{d \tau} & =T(\phi)-\phi \\
& =\left(\begin{array}{c}
\frac{\alpha+\mu a_{1}\left(\beta_{0}+\beta_{1}+\beta_{1}(1-\mu) b_{2}\right)+(1-\mu) a_{2}\left(\beta_{0}+\beta_{1}+\beta_{1}(1-\mu) b_{2}\right)}{1-(1-\mu) b_{2}\left(\beta_{0}+(1-\mu) \beta_{1} b_{2}\right)}-a_{1} \\
\alpha+\mu a_{1}\left(\beta_{0}+\beta_{1}+\beta_{1}(1-\mu) b_{2}\right)+(1-\mu) a_{2}\left(\beta_{0}+\beta_{1}+\beta_{1}(1-\mu) b_{2}\right)-a_{2} \\
(1-\mu) b_{2}\left(\beta_{0}+(1-\mu) \beta_{1} b_{2}\right)-b_{2}
\end{array}\right.
\end{aligned}
$$

To check for stability, we must find the Jacobian matrix of the previous ODE and look at the eigenvalues of that matrix. I find that the eigenvalues of this matrix are:

Eigenvalues of Jacobian=

$$
\begin{gathered}
-1 \\
(1-\mu) \beta_{0}+(1-\mu)^{2} 2 \beta_{1} b_{2}-1 \\
\frac{\beta_{1}-\left(\beta_{0}+(1-\mu) b_{2} \beta_{1}\right)\left(-1-(1-\mu) b_{2}\left(1-(1-\mu) \beta_{0}+(1-\mu)\left(-1-(1-\mu) b_{2}\right) \beta_{1}\right)\right)-1}{\left(1-(1-\mu) b_{2}\left(\beta_{0}+(1-\mu) b_{2} \beta_{1}\right)\right)}
\end{gathered}
$$

All these eigenvalues must have negative real parts. Note that the third eigenvalue has a restriction that the denominator can not be equal to zero.

\subsection{Calculation of the MSE for both of the PLM's}

\section{MSE for the first PLM}

PLM1:

$$
\begin{aligned}
M S E_{1}= & E\left(y-a_{1}\right)^{2} \\
= & E\left(T_{a 2}+T_{b 2} y_{t-1}+v_{t}-a_{1}\right)^{2} \\
= & E\left(T_{a 2}^{2}\right)+E\left(T_{b 2}^{2} y_{t-1}^{2}\right)+E\left(v_{t}^{2}\right)+E\left(a_{1}^{2}\right)+2 E\left(T_{a 2} T_{b 2} y_{t-1}\right) \\
& -2 E\left(T_{a 2} a_{1}\right)-2 E\left(a_{1} T_{b 2} y_{t-1}\right) \\
= & a_{2}^{2}+b_{2}^{2} E\left(y_{t-1}^{2}\right)+\sigma_{v}^{2}+a_{1}^{2}+2 a_{2} b_{2} a_{1}-2 a_{1} a_{2}-2 a_{1}^{2} b_{2} \\
= & a_{2}^{2}+\frac{b_{2}^{2}}{1-b_{2}^{2}}\left(a_{2}^{2}+2 a_{1} a_{2} b_{2}+\sigma_{v}^{2}\right)+\sigma_{v}^{2}+a_{1}^{2}+2 a_{1} a_{2} b_{2}-2 a_{1} a_{2}-2 a_{1}^{2} b_{2} \\
= & \left(a_{2}^{2}+2 a_{1} a_{2} b_{2}^{3}+\sigma_{v}^{2}\right) \frac{1}{1-b_{2}^{2}}+a_{1}^{2}+2 a_{1} a_{2} b_{2}-2 a_{1} a_{2}-2 a_{1}^{2} b_{2}
\end{aligned}
$$

If $b=0$ then the MSE from the first predictor becomes:

$$
M S E_{1}=\sigma_{v}^{2}
$$


When we enter the MEE values in for the $M S E_{1}$ we get the following solution:

$$
M S E_{1}=\frac{(1-\mu)^{4} \sigma^{2} \beta_{1}^{2}}{(1-\mu)^{4} \beta_{1}^{2}-\left(1-(1-\mu) \beta_{0}\right)^{2}}
$$

The $M S E_{1}$ is not defined at all values since the denominator may be zero at some values of $\mu, \beta_{0}$, and $\beta_{1}$. Next, the denominator is solved for zero to see where the $M S E_{1}$ is undefined.

$$
\begin{gathered}
(1-\mu)^{4} \beta_{1}^{2}-\left(1-(1-\mu) \beta_{0}\right)^{2}=0 \\
\Rightarrow\left((1-\mu)^{2} \beta_{1}-1+(1-\mu) \beta_{0}\right)\left((1-\mu)^{2} \beta_{1}+1-(1-\mu) \beta_{0}\right)=0
\end{gathered}
$$

Here are both solutions to the above equation:

$$
\begin{aligned}
& \Rightarrow \quad(1-\mu)\left(\beta_{0}+(1-\mu) \beta_{1}\right)=1 \\
& \Rightarrow \quad \beta_{1}=\frac{1}{(1-\mu)^{2}}-\frac{1}{(1-\mu)} \beta_{0}
\end{aligned}
$$

and

$$
\begin{aligned}
& \Rightarrow \quad(1-\mu)\left((1-\mu) \beta_{1}-\beta_{0}\right)=-1 \\
& \Rightarrow \quad \beta_{1}=-\frac{1}{(1-\mu)^{2}}+\frac{1}{(1-\mu)} \beta_{0}
\end{aligned}
$$

The above equations describe the values where the model goes from stationary to non-stationary. If we just concern ourselves with values that are stationary, then we will not have to worry about the $M S E_{1}$ being undefined.

\section{MSE for the second PLM}

PLM2:

$$
\begin{aligned}
M S E_{2}= & E\left(y-a_{2}-b_{2} y_{t-1}\right)^{2} \\
= & E\left(T_{a 2}+T_{b 2} y_{t-1}+v_{t}-a_{2}-b_{2} y_{t-1}\right)^{2} \\
= & a_{2}^{2}+b_{2}^{2} E\left(y_{t-1}^{2}\right)+\sigma_{v}^{2}+a_{2}^{2}+b_{2}^{2} E\left(y_{t-1}^{2}\right)+2 a_{1} a_{2} b_{2}-2 a_{2}^{2}-2 a_{1} a_{2} b_{2} \\
& -2 a_{1} a_{2} b_{2}-2 b_{2}^{2} E\left(y_{t-1}^{2}\right)+2 a_{1} a_{2} b_{2} \\
= & \sigma_{v}^{2}
\end{aligned}
$$

The mean square error for the second predictor will always be $\sigma_{v}^{2}$ as long as y follows a stationary process. This means that the $M S E_{1} \geq M S E_{2}$ for all E-stable stationary values of $\alpha, \beta_{0}$, and $\beta_{1}$. This intuitively makes sense because the $\operatorname{AR}(1)$ predictor is always unbiased while the MSV predictor is unbiased only when $b_{2}=0$. 


\section{References}

[1] Azariadis, C., 1981. Self-Fulfilling Prophecies. Journal of Economic Theory 25, 380-396.

[2] Azariadis, C., Guesnerie, R., 1986 Sunspots and Cycles. Review of Economic Studies 53, 725-737.

[3] Bray, M., Savin, N.E., 1986. Rational Expectations Equilibria, Learning, and Model Specification. Econometrica 54, 1129-1160.

[4] Cass, D., Shell, K., 1983 Do Sunspots Matter. Journal of Political Economy 91, 193-227.

[5] Evans, G.W., 1989. The Fragility of Sunspots and Bubbles. Journal of Monetary Economics 23, 297-317.

[6] Evans, G.W., Honkapohja, S., 1992. On the Robustness of Bubbles in Linear RE Models. International Economic Review 33, 1-14.

[7] Evans, G.W., Honkapohja, S., 1994. Learning, Convergence, and Stability with Multiple Rational Expectations Equilibria. European Economic Review 38, 1071-1098.

[8] Evans, G.W., Honkapohja, S., 1997. Least Squares Learning with Heterogenous Expectations. Economic Letters 52, 197-201.

[9] Evans, G.W., Honkapohja, S., 2001. Learning and Expectations in Macroeconomics. Princeton University Press, Princeton, NJ.

[10] Evans, G.W., Honkapohja, S., Marimon, R., 2001. Convergence in Monetary Inflation Models with Heterogenous Learning Rules. Macroeconomic Dynamics 5, 1-31.

[11] Farmer, R., 1999. Macroeconomics Self-fulfilling Prophecies. MIT Press, Cambridge, MA.

[12] Giannitsarou, C., 2003. Heterogeneous Learning. Review of Economic Dynamics (forthcoming).

[13] Guesnerie, R., 1992. An Exploration of the Eductive Justifications of the Rational Expectations Hypothesis. American Economic Review 82, 1254-1278.

[14] Heinemann, M., 2000. Convergence of Adaptive Learning and the Validity of the E-stability Principle. Macroeconomic Dynamics 4, 263-288.

[15] Hommes, C., Sorger, G., 1998. Consistent Expectations Equilibria. Macroeconomic Dynamics 2, 287-321. 
[16] Honkapohja, S., Mitra, K. 2002. Learning Stability in Economics with Heterogeneous Agents. Mimeo.

[17] Ljung, L., 1977. Analysis of Recursive Stochastic Algorithms. IEEE Transactions on Automatic Control 22, 551-575.

[18] Lucas, R.E., Jr., 1972. Expectations and the Neutrality of Money. Journal of Economic Theory 4, 103-124.

[19] Lucas, R.E., Jr., 1973. Some International Evidence on the Output-Inflation Trade-offs. American Economic Review 63, 326-334.

[20] Marcet, A., Sargent, T.J., 1989. Convergence of Least Squares Learning and the Dynamic of Hyperinflation, in: Barnett, W., Geweke, J., Shell, K. (Eds.), Economic Complexity: Chaos, Sunspots, Bubbles, and Nonlinearity, Cambridge University Press, Cambridge, 119-137.

[21] Muth, J. F., 1961. .Rational Expectations and the Theory of Price Movements. Econometrica 29, 315-335.

[22] Sargent, T. J., Wallace, N., 1975. "Rational" Expectations, the Optimal Monetary Instrument and the Optimal Money Supply Rule. Journal of Political Economy 83, 241-254.

[23] Taylor, J., 1977. Conditions for Unique Solutions in Stochastic Macroeconomic Models with Rational Expectations. Econometrica 45, 1377-1386.

[24] Townsend, R., 1978. Market Anticipations, Rational Expectations, and Bayesian Analysis. International Economic Review 19, 481-494. 